\title{
Energy-Based Criterion for Testing the Nonlinear Response Strength of Strong Nonlinear Oscillators
}

\author{
BIG-ALABO, AKURO \\ Applied Mechanics \& Design (AMD) Research Group, Department of Mechanical Engineering, Faculty of Engineering, University of Port \\ Harcourt, Port Harcourt, Nigeria \\ ${ }^{a}$ Corresponding Email: akuro.big-alabo@uniport.edu.ng
}

\begin{abstract}
This article proposes a simple energy-based criterion developed to characterize four commonly identified responses, namely: linear, weakly nonlinear, moderately nonlinear and strongly nonlinear regimes. The response of the nonlinear simple pendulum was used for benchmarking the boundary conditions for each of the four response regimes and the test criterion was demonstrated using relevant examples. The test presented in this article is important for clarifying the obscurity surrounding the accuracy and range of validity of recent approximate analytical schemes used to investigate strong nonlinear oscillators. Furthermore, it is meant to create awareness of the need to develop more robust testing criteria.
\end{abstract}

\section{DOI: https://dx.doi.org/10.4314/jasem.v25i2.14}

Copyright: Copyright (C) 2021 Akuro, Big-Alabo. This is an open access article distributed under the Creative Commons Attribution License (CCL), which permits unrestricted use, distribution, and reproduction in any medium, provided the original work is properly cited.

Dates: Received: 12 December 2020; Revised: 26 January 2021; Accepted: 12 February 2021

Keywords: strong nonlinear oscillation; periodic oscillation; approximate analytical solution

The study of nonlinear oscillations is important to engineers, physicists and applied mathematicians. In the last three decades, a lot of attention has been devoted to the formulation of accurate schemes for the solution of nonlinear oscillators. To this end, a 'flood' of approximate analytical methods for the periodic solution of nonlinear oscillators have appeared in the literature (see the publications by $\mathrm{He}$ (2006), Esmailzadeh et al (2019) and Big-Alabo and Ossia (2020) for discussions on various recent methods). All of these methods claim to address some or all of the following limitations: (i) Lack of physical insight into the dynamic response of oscillating systems, which is mainly a limitation of the numerical methods. (ii) Algebraic complexity, which is a limitation of methods requiring high-order approximations. (iii) Inaccurate solution for strong nonlinear and largeamplitude oscillations, which is a limitation of traditional perturbation methods. (iv) Use of artificial parameters that have no physical meaning, which is a limitation of traditional and recent perturbation methods. (v) Use of truncated Taylor's series to represent non-polynomial restoring force, which is a limitation of series expansion methods including all perturbation methods. A good proportion of these methods address limitations $1,2,4$ and 5 to various degrees, but the problem lies in limitation 3 where a disturbing trend seems to occur. The issue is that many studies apply approximate analytical methods to nonlinear oscillators aimed at providing solutions for the strong nonlinear response, but the results presented seem to be carefully chosen so as to portray the analytical solutions as accurate. A close scrutiny of the results usually reveal that the analytical solutions were only tested for linear or weakly to moderately nonlinear cases (Sanchez, 2005); whereas if the solutions were tested for strong nonlinear cases, they produced large errors and failed to capture essential nonlinear behaviour (Sanchez, 2005; Big-Alabo, 2018a). This article therefore presents a simple energy-based criterion that can be used to test the nonlinear strength of an oscillator based on its amplitude and system parameters. The criterion was calibrated using the simple pendulum and tested for some strong nonlinear oscillators commonly found in the literature.

\section{MATERIALS AND METHOD}

Nonlinear conservative oscillators can be represented in general form as (Big-Alabo, 2018a):

$$
\ddot{u}+g(u)=0 \quad 1
$$

Where $g(u)$ is a nonlinear restoring force, the initial conditions are $u(0)=A$ and $\dot{u}(0)=0$, and $A$ is the 
amplitude of oscillation. The maximum potential energy is then given as:

$$
V=\int_{0}^{A} g(u) d u \quad 2
$$

The present criterion is based on comparing equation (2) with the energy of an equivalent linear oscillator given as:

$$
\ddot{u}+K_{e q} u=0 \quad 3
$$

where $K_{e q}=g(A) / A$ was obtained as illustrated in Figure 1 and the initial conditions are the same as in equation (1). Therefore,

$$
V_{e q}=\frac{1}{2} K_{e q} A^{2}=\frac{1}{2} A g(A) \quad 4
$$

Since $V_{e q}$ is an approximation, its relative error can be calculated as:

$$
\epsilon_{r}=\left|1-\frac{V_{e q}}{V}\right| \times 100 \%=\left|1-\frac{A g(A)}{2 \int_{0}^{A} g(u) d u}\right| \times 100 \% 5
$$

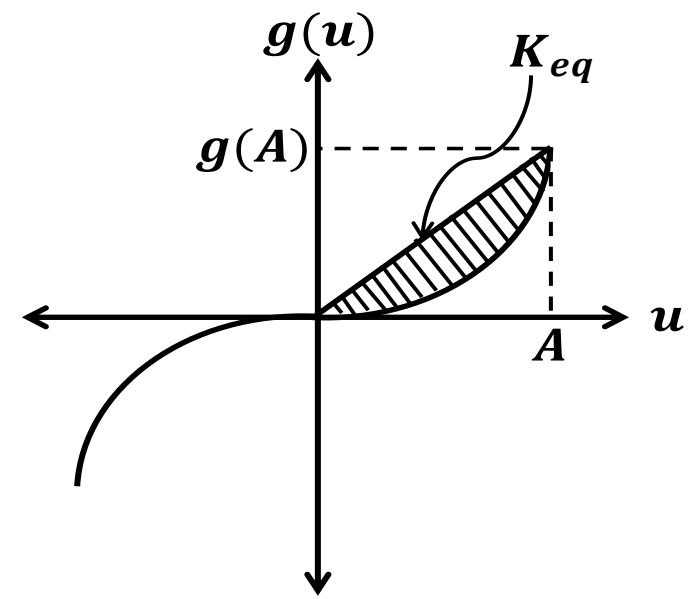

Fig 1: Restoring force of a typical nonlinear oscillator with equivalent linear force showing error of linearization (shaded portion)

To develop the test criterion, four response regimes with various degrees of nonlinear strength were considered, namely: (a) linear (b) weakly nonlinear (c) moderately nonlinear and (d) strong nonlinear. Equation (5) can be used to determine which of the four response regimes a nonlinear oscillator would exhibit for a given set of input values. In setting the boundaries of $\epsilon_{r}$ for each regime, we calibrate using the simple pendulum. The simple pendulum was chosen because it can exhibit any of the four response regimes and there is a general consensus of what these regimes are for the simple pendulum. So, $A \leq 10^{\circ}=$ $\pi / 18 \mathrm{rad}$ represents the linear response regime, $10^{\circ}<A \leq 30^{\circ}=\pi / 6 \mathrm{rad}$ represents the weakly nonlinear response regime, $30^{\circ}<A \leq 90^{\circ}=\pi /$
$2 \mathrm{rad}$ represents the moderately nonlinear response regime, and $90^{\circ}<A<180^{\circ}=\pi \mathrm{rad}$ represents the strong nonlinear response regime. The restoring force for the simple pendulum is $g(u)=\omega_{0}^{2} \sin u$ where $\omega_{0}=\sqrt{g / l}, g$ is the local acceleration due to gravity and $l$ is the length of the pendulum. Hence, from equation (5) we get:

$$
\epsilon_{r}=\left|1-\frac{A \sin A}{2(1-\cos A)}\right| \times 100 \% \quad 6
$$

Applying equation (6) and the above amplitude range for each response regime, the following are applicable to a general nonlinear oscillator: (i) Linear response regime: $\epsilon_{r} \leq 0.25 \%$ (ii) Weak nonlinear response regime: $0.25 \%<\epsilon_{r} \leq 2.5 \%$ (iii) Moderate nonlinear response regime: $2.5 \%<\epsilon_{r} \leq 25 \%$ (iv) Strong nonlinear response regime: $\epsilon_{r}>25 \%$

The boundaries of $\epsilon_{r}$ for each response regime are not strict and may overlap depending on the case considered, but they are meant to provide some objective determination of the strength of a nonlinear response. Hence, for a given set of system parameters, the amplitude that would give a particular $\epsilon_{r}$ can be obtained by solving the following nonlinear equation:

$$
A g(A)+2\left(0.01 \epsilon_{r}-1\right) \int_{0}^{A} g(u) d u=0 \quad 7
$$

\section{RESULTS AND DISCUSSIONS}

To demonstrate the applicability of the energy-based criterion, a number of strong nonlinear oscillators capable of exhibiting each of the four response regimes were investigated. The selected oscillators cover a range of nonlinear oscillators including oddly nonlinear oscillators, parameter-dependent systems, non-natural systems and asymmetric oscillators. The purpose of the investigation is to apply the present test criterion to determine the response regimes for a given set of input and to evaluate the accuracy of published solutions for strong nonlinear responses.

Electrostatically-actuated nanobeam with weak interacting forces: The nonlinear vibration of an electrostatically-actuated nanobeam with van der Waals, Casimir and fringing effects can be modelled as (Ghalambaz et al, 2016):

$$
\begin{aligned}
& \left(a_{2}-4 a_{4} u^{2}+6 a_{6} u^{4}-4 a_{8} u^{6}+a_{10} u^{8}\right) u^{\prime \prime}+ \\
& \left(E_{1} u+E_{2} u^{3}+E_{3} u^{5}+E_{4} u^{7}+E_{5} u^{9}+E_{6} u^{11}\right)=0 \\
& 8
\end{aligned}
$$

where $a_{2}, a_{4}, a_{6}, a_{8}, a_{10}, E_{1}, E_{2}, E_{3}, E_{4}, E_{5}$, and $E_{6}$ are constants depending on the system properties and $u$ is the dimensionless displacement. Comparing equations (8) and (1), we can write that: 


$$
g(u)=\frac{E_{1} u+E_{2} u^{3}+E_{3} u^{5}+E_{4} u^{7}+E_{5} u^{9}+E_{6} u^{11}}{a_{2}-4 a_{4} u^{2}+6 a_{6} u^{4}-4 a_{8} u^{6}+a_{10} u^{8}}
$$

Therefore, using equation (5) gives:

$$
\epsilon_{r}=\left|1-\frac{\left(\frac{E_{1} A^{2}+E_{2} A^{4}+E_{3} A^{6}+E_{4} A^{8}+E_{5} A^{10}+E_{6} A^{12}}{a_{2}-4 a_{4} A^{2}+6 a_{6} A^{4}-4 a_{8} A^{6}+a_{10} A^{8}}\right)}{2 \int_{0}^{A}\left(\frac{E_{1} u+E_{2} u^{3}+E_{3} u^{5}+E_{4} u^{7}+E_{5} u^{9}+E_{6} u^{11}}{a_{2}-4 a_{4} u^{2}+6 a_{6} u^{4}-4 a_{8} u^{6}+a_{10} u^{8}}\right) d u}\right| \times
$$

The integral in equation (10) does not have a closedform solution and was evaluated numerically. The energy profiles of the original and equivalent linear models are shown in Figure $2 \mathrm{a}$ for the following input values: $\quad \delta=0.50 ; \alpha_{c a}=25.0 ; \alpha_{v d w}=25.0 ; \alpha=$ 24.0.; $\beta=25.0$; ? $=0.65 ; N=10.0$. The range of amplitudes for which the nanobeam can undergo oscillations can be obtained by solving $g(A)=0$. For the input values used in Figure $2 \mathrm{a}$, the amplitude range was calculated as $0<A \leq 0.585$. The error analysis of Figure $2 \mathrm{~b}$ showed that the nanobeam undergoes a linear to weakly nonlinear response for $0<A<0.35$, while a strong nonlinear response was predicted when $A>0.475$.
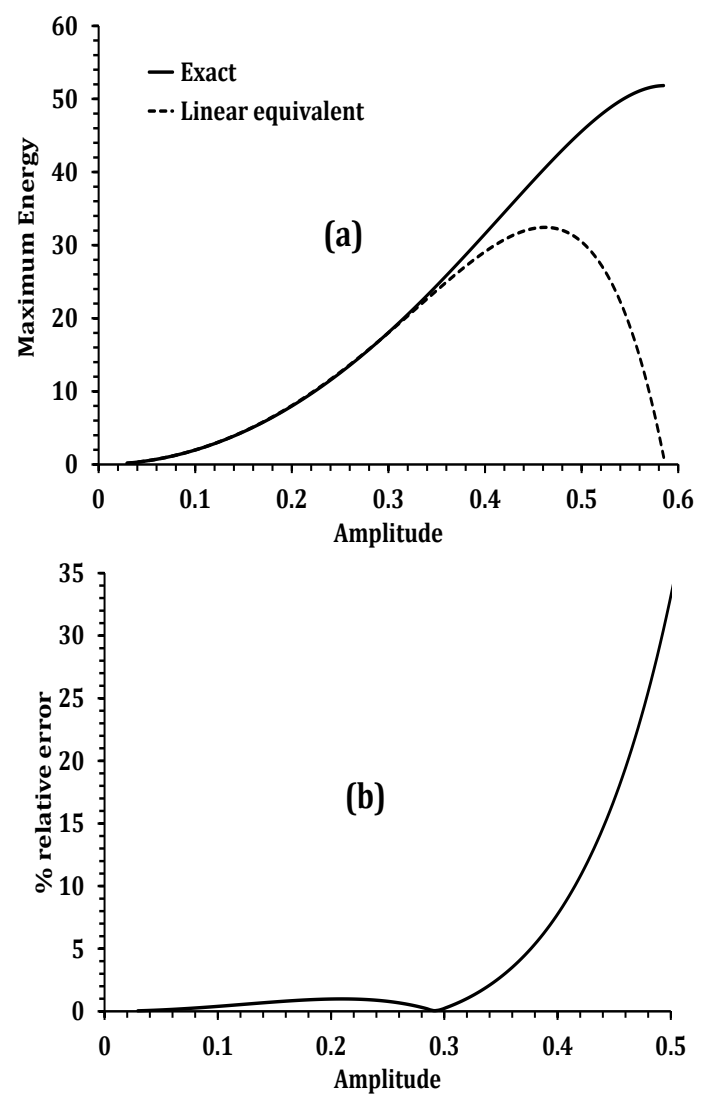

Fig 2: (a) Energy profile of a nanobeam with weak interacting forces and (b) error estimate for the equivalent linear approximation.
Equations (8) was studied by Ghalambaz et al (2016) using the energy balance method (EBM) while Ismail et al (2019) used the global residue harmonic balance method (GRHBM). The EBM solution was simulated for $A=0.01$ while the GRHBM was applied for $A \leq$ 0.50 . The present criterion shows that $A=0.01$ is in the linear response regime while $A=0.50$ is in the strong nonlinear regime. It was observed that the error in the frequency estimate of the GRHBM increased with amplitude and exceeded $5.0 \%$ relative error at $A=0.40$ while the EBM exceeded $5.0 \%$ relative error at $A=0.30$. Hence, the EBM and GRHBM solutions are not accurate for predicting the strong nonlinear oscillations of the electrostatically-actuated nanobeam with weak interacting forces.

Relativistic oscillator: The simple harmonic relativistic oscillator is a relativistic mass that is connected to a linear spring. For non-relativistic speeds, the mass exhibits a simple harmonic motion with a constant period. For relativistic speeds, the mass exhibits a nonlinear response with amplitudedependent period and anharmonic oscillation history. The nonlinearity in the system is due to the relativistic effect. The model for the relativistic oscillator is given as (Big-Alabo, 2018b):

$$
\ddot{u}+\omega_{0}^{2}\left[1-\left(\frac{\dot{u}}{c}\right)^{2}\right]^{3 / 2} u=0
$$

with initial conditions $u(0)=0$ and $\dot{u}(0)=V_{0}$, where $V_{0}$ is the initial velocity, $c=3 \times 10^{8} \mathrm{~m} / \mathrm{s}$ is the speed of light, $\omega_{0}=\sqrt{K_{L} / m_{0}}, K_{L}$ is the stiffness of the linear spring and $m_{0}$ is the rest mass. Using the nondimensional parameters, $U=u \omega_{0} / c$ and $\tau=\omega_{0} t$, equation (11) can be expressed as:

$$
U^{\prime \prime}+\left[1-\left(U^{\prime}\right)^{2}\right]^{3 / 2} U=0 \quad 12
$$

where the prime denotes differentiation with respect to $\tau$ and the initial conditions are $U(0)=0$ and $U^{\prime}(0)=$ $\beta_{0}=V_{0} / c$. Equation (12) can be expressed in the form of equation (1) as shown:

$$
U^{\prime \prime}+\left[\frac{1}{\sqrt{1-\beta_{0}^{2}}}-\frac{1}{2} U^{2}\right]^{-3} U=0
$$

where the dimensionless restoring force is:

$$
g(U)=\left[\frac{1}{\sqrt{1-\beta_{0}^{2}}}-\frac{1}{2} U^{2}\right]^{-3} U
$$


The initial conditions to equation (13) are the same as equation (1) and the dimensionless amplitude is calculated as:

$$
A=\left[\frac{2\left(1-\sqrt{1-\beta_{0}^{2}}\right)}{\sqrt{1-\beta_{0}^{2}}}\right]^{1 / 2} \quad 15
$$

Therefore, substituting equations (14) and (15) into equation (5) gives,

$$
\epsilon_{r}=\left|1-\frac{2\left(1-\sqrt{1-\beta_{0}^{2}}\right)}{\beta_{0}^{2} \sqrt{1-\beta_{0}^{2}}}\right| \times 100 \%=\left|1-\left(\frac{A}{\beta_{0}}\right)^{2}\right| \times
$$$$
100 \% \quad 16
$$
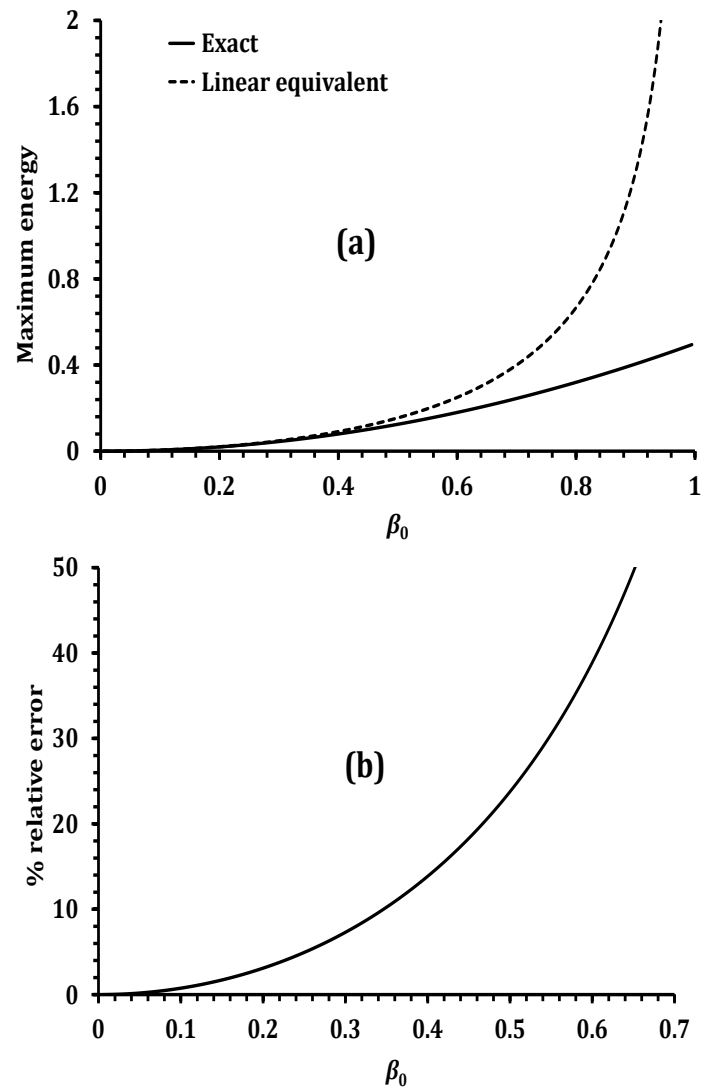

Fig 3: (a) Energy profile of the simple harmonic relativistic oscillator and (b) error estimate for the equivalent linear approximation.

As seen from Figure 3a, the energy predicted by the equivalent linear model is always higher than that of the corresponding original model and the error estimate in Figure $3 \mathrm{~b}$ increases rapidly with increase in $\beta_{0}$ due to the highly nonlinear nature of the oscillator. A linear to weakly nonlinear response occurs for $0<\beta_{0}<0.185$, while a strong nonlinear response occurs when $\beta_{0}>0.51$. Hence, the studies conducted on the relativistic oscillator by Ebaid (2010) and Baizar and Hosami (2014) are for weakly nonlinear response.
Tapered beam with static and inertia nonlinearity: The free vibration of a tapered beam with static and inertia nonlinearities has been widely investigated and the governing model is given as (Baizar and Hosami, 2014):

$$
\ddot{u}+u+\alpha u^{4} \ddot{u}+2 \alpha u^{3} \dot{u}^{2}+\beta u^{5}=0
$$

Equation (17) is a non-natural conservative oscillator leading to an exact differential equation and can be transformed into the form of equation (1) using the procedure in (Big-Alabo, 2020). Hence,

$$
\ddot{u}+\frac{2 \alpha u^{3}\left[\left(A^{2}-u^{2}\right)+\beta\left(A^{6}-u^{6}\right) / 3\right]}{\left(1+\alpha u^{4}\right)^{2}}+\frac{u+\beta u^{5}}{\left(1+\alpha u^{4}\right)}=0
$$

Where

$$
g(u)=\frac{2 \alpha u^{3}\left[\left(A^{2}-u^{2}\right)+\beta\left(A^{6}-u^{6}\right) / 3\right]}{\left(1+\alpha u^{4}\right)^{2}}+\frac{u+\beta u^{5}}{\left(1+\alpha u^{4}\right)}
$$

This implies that:

$$
\epsilon_{r}=\left|1-\frac{3\left(1+\beta A^{4}\right)}{\left(3+\beta A^{4}\right)\left(1+\alpha A^{4}\right)}\right| \times 100 \%
$$

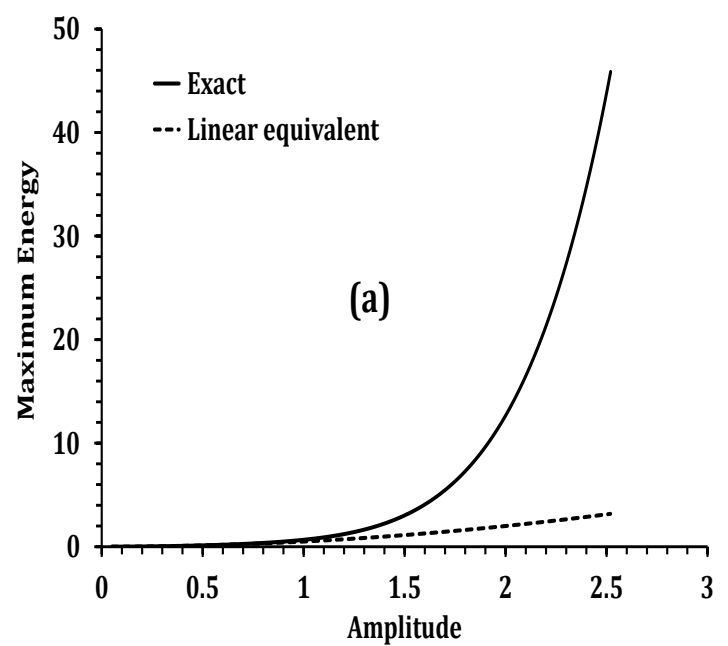

Fig 4: (a) Energy profile of a tapered beam with static and inertia nonlinearities and

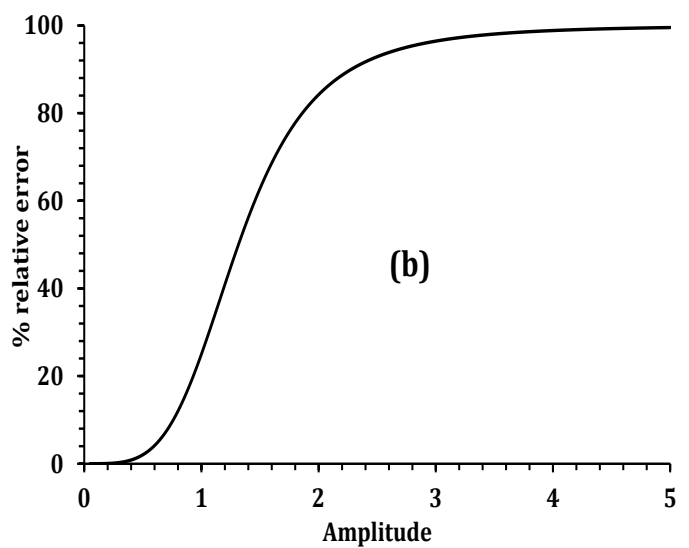

Fig 4: (b) error estimate for the equivalent linear approximation $(\alpha=\beta=1.0)$ 
The energy profile in Figure 4a and the error analysis in Figure $4 \mathrm{~b}$ shows that the system exhibits linear to weakly nonlinear response for $A<0.53$ while a strong nonlinear response was observed for $A \geq 1.0$. Ismail (2017) studied this tapered beam model using a coupled homotopy-variational approach (CHVA). The study focussed on the strong nonlinear regime and the solution derived for the natural frequency produced more than $1.0 \%$ error when $A<50$. On the other hand, the error in the natural frequency estimate reduced to zero when $A \geq 100$. In spite of the fact that the CHVA gave accurate frequency-amplitude response for $A \geq 100$, the estimated displacement profile shows large deviations from the actual displacement (Big-Alabo et al, 2020). This shows that the CHVA is inaccurate for the strong nonlinear periodic oscillations of the tapered beam.

Stretched elastic cable with mid-point mass: The vibration of a mid-point mass attached to a stretched elastic cable has been studied by many (Big-Alabo, 2019b; Elias-Zuniga, 2014; Belendez et al, 2009). Its model is given as (Big-Alabo, 2019b):

$$
\ddot{u}+\omega_{0}^{2}\left(u-\frac{\psi u}{\sqrt{u^{2}+1}}\right)=0
$$

This oscillator exhibits a linear or weakly nonlinear response for $A<0.10$ and $A>10.0$ when $\psi<1.0$. The moderate to strong nonlinear response occurs in the amplitude range of $0.10<A<10.0$. Hence, it is easy to test a solution method outside this range and conclude that the method works for the strong nonlinear oscillations. Substituting the restoring force of equation (21) in (5) gives:

$$
\epsilon_{r}=\left|1-\frac{A^{2}\left(1-\frac{\psi}{\sqrt{A^{2}+1}}\right)}{\left[A^{2}+2\left(1-\sqrt{A^{2}+1}\right) \psi\right]}\right| \times 100 \%
$$

Since the nonlinear response of this oscillator depends on $\psi$, the energy profile and error analyses were conducted for $\psi=[0.1,0.5,0.9]$ as shown in Figure $5 \mathrm{a}-\mathrm{d}$. The plots showed that the response exhibits weakly or moderately nonlinear response for all amplitudes when $\psi<0.75$. This explains why only $\psi>0.9$ showed the presence of strong nonlinear response for certain amplitudes i.e. $0.425 \leq A \leq 3.0$.

Asymmetric quadratic nonlinear oscillator: So far, all the oscillators considered are symmetric (i.e. oddly nonlinear) and equation (5) applies to such oscillators. Here we consider asymmetric oscillators in which the amplitude is different for each half-cycle.
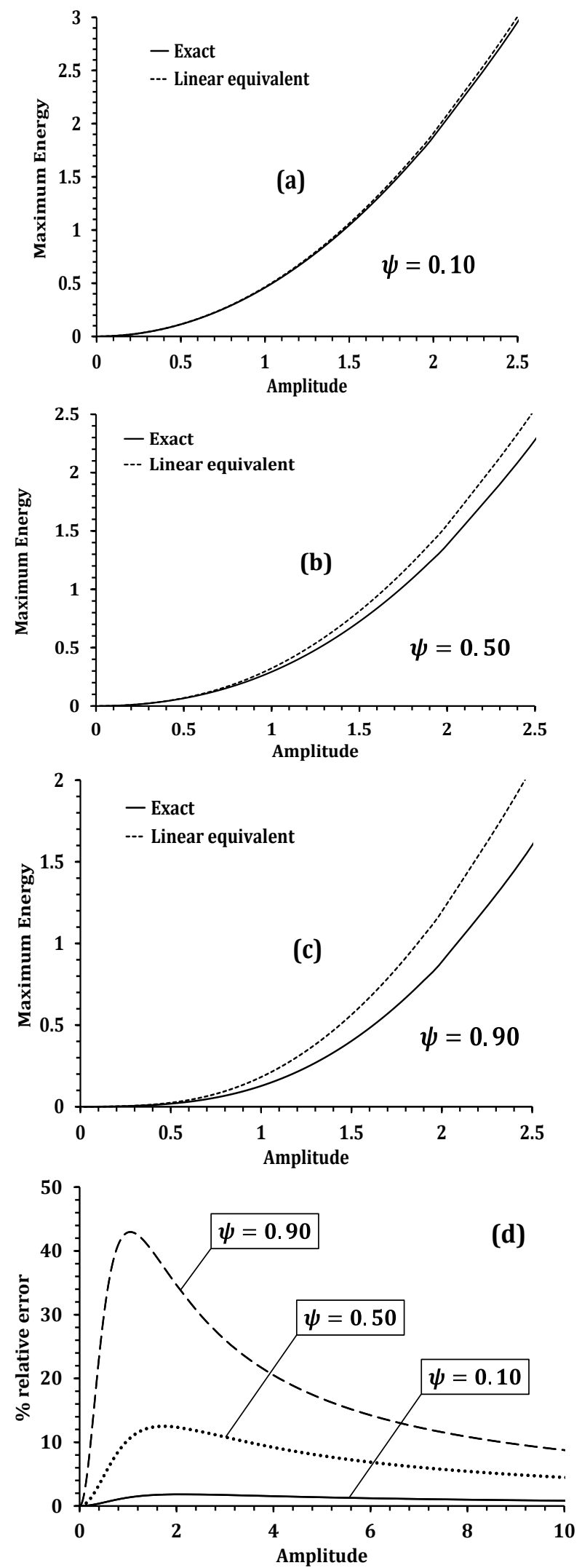

Fig 5: (a-c) Energy profile of stretched elastic cable with mid-point mass and (d) error estimate for the equivalent linear approximation. 
Examples of asymmetric oscillators include the quadratic nonlinear oscillator, Helmholtz-Duffing oscillator and nonlinear ship roll motion. For these oscillators, the potential energy is determined from $A$ to $-B$ where $A$ and $B$ are the amplitudes on each halfcycle and $A \neq B$.

This implies that a bi-linear approximation would be applied. Therefore,

$$
K_{e q}=\left\{\begin{array}{lr}
g(A) / A & 0<u<A \\
g(B) / B & 0>u>-B
\end{array}\right.
$$

and

$$
V_{e q}=\frac{1}{2}[A g(A)+B g(B)] \quad 24
$$

So that $\epsilon_{r}$ for an asymmetric oscillator can be determined as:

$$
\epsilon_{r}=\left|1-\frac{A g(A)+B g(B)}{2\left(\int_{0}^{A} g(u) d u+\int_{0}^{-B} g(u) d u\right)}\right| \times 100 \%
$$

The same boundaries of $\epsilon_{r}$ defined in section 2 are applicable for the asymmetric oscillators.
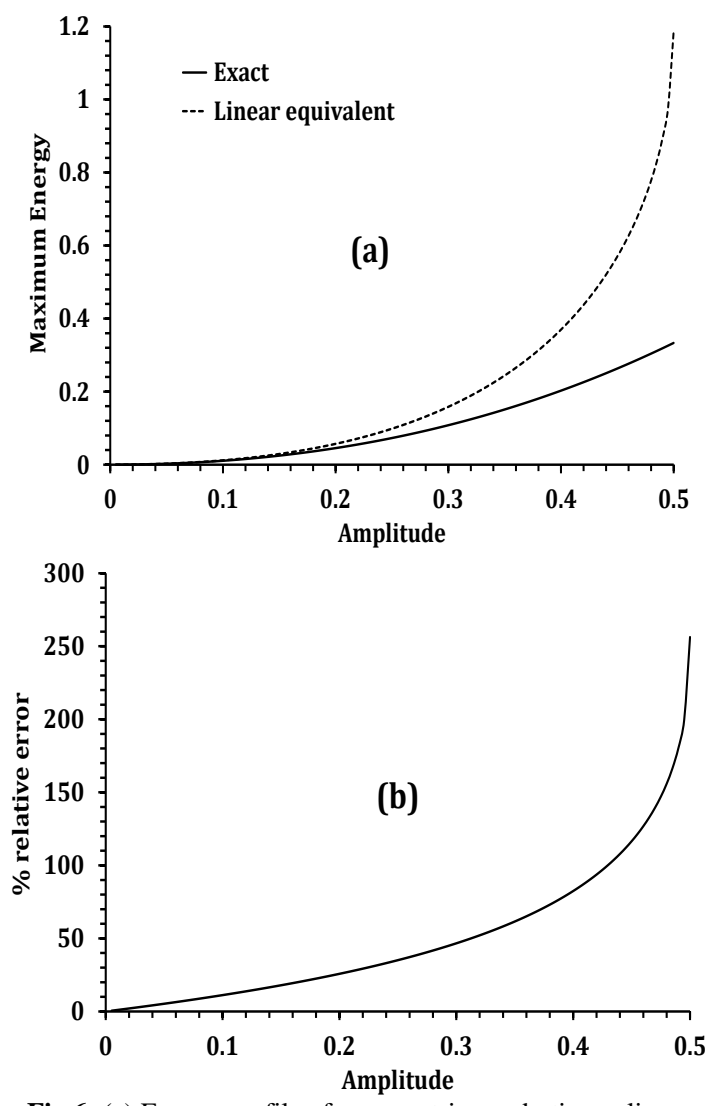

Fig 6: (a) Energy profile of asymmetric quadratic nonlinear oscillator and (b) error estimate for the equivalent linear approximation
The model for the asymmetric quadratic nonlinear oscillator can be expressed as:

$$
\ddot{u}+u+u^{2}=0 \quad 26
$$

where $g(u)=u+u^{2}$, the initial conditions are $u(0)=A$ and $\dot{u}(0)=0$, and the system oscillates in the range $u \in[A,-B]$. It can be easily demonstrated that the initial amplitude for which periodic oscillations are possible is $0<A \leq 1 / 2$. Also, the amplitude on the other half-cycle can be derived as:

$$
B=\frac{2 A+3-\sqrt{(1-2 A)(9+6 A)}}{4}>A
$$

Hence, the corresponding limit of $B$ is $0<B \leq 1$. Consequently, $\epsilon_{r}$ can be derived as:

$$
\epsilon_{r}=\left|1-\frac{A^{2}(1+A)+B^{2}(1+B)}{\left(A^{2}+B^{2}\right)+2\left(A^{3}-B^{3}\right) / 3}\right| \times 100 \%
$$

Figure 6a shows that the total potential energy of the equivalent bi-linear oscillator is always greater than the exact total potential energy. The corresponding error analysis in Figure $6 \mathrm{~b}$ suggests that the system exhibits a weakly nonlinear response when $A<0.025$ and a strong nonlinear response when $A>0.2$.

Conclusion: In this article, a simple energy-based criterion was developed to estimate the strength of the nonlinearity of an oscillator for any given set of input parameters. Given that there is no known prior study of this nature, the present study is an attempt to categorize the response of conservative nonlinear oscillators so that solution schemes developed to provide periodic solutions can be properly evaluated. It is hoped that this study will generate interest in nonlinear scientists to develop a more rigorous test criterion for the same purpose. The present criterion is based on simple energy considerations and provides a quick test.

\section{REFERENCES}

Baizar, J and Hosami, M (2014). An easy trick to a periodic solution of relativistic harmonic oscillator. J. Egypt. Math. Soc. 22: 45 - 49.

Belendez, A; Alvarez, ML; Fernandez, E; Pascual, I (2009). Cubication of conservative nonlinear oscillators. Eur. J. Phy. 30(5): 973-981.

Big-Alabo A (2018b). Continuous piecewise linearization method for approximate periodic solution of the relativistic oscillator. Int. J. Mech. Eng. Educ. DOI: 10.1177/0306419018812861. 
Big-Alabo, A (2018a). Periodic solutions of Duffingtype oscillators using continuous piecewise linearization method, Mech. Eng. Res. 8(1): 41-52.

Big-Alabo, A (2019a). Approximate periodic solution for the large-amplitude oscillations of a simple pendulum. Int. J. Mech. Eng. Educ., DOI: 10.1177/0306419019842298.

Big-Alabo, A (2019b). A simple cubication method for approximate solution of nonlinear Hamiltonian oscillators. Int. J. Mech. Eng. Educ., DOI: $10.1177 / 0306419018822489$.

Big-Alabo, A (2020). Approximate periodic solution and qualitative analysis of non-natural oscillators based on the restoring force. Eng. Res. Exp. 2(1): $14 \mathrm{pp}$

Big-Alabo, A; Ossia, CV (2020). Periodic Solution of Nonlinear Conservative Systems. In: Buzea, CG; Agop, M; Butler, L (eds) Progress in Relativity. IntechOpen, UK, DOI: 10.5772/intechopen.90282.

Big-Alabo, A; Ossia, CV; Ekpruke, EO; Ogbonnia, CD (2020). Large-amplitude vibration analysis of a strong nonlinear tapered beam using continuous piecewise linearization method. J. King Saud Uni. Eng. Sci. DOI:10.1016/j.jksues.2020.11.005.

Ebaid, AE (2010). Approximate periodic solutions for the non-linear relativistic harmonic oscillator via differential transformation method. Comm. Nonlinear Sci. and Num. Sim. 13: 1921-1927.

Elias-Zuniga, A (2014). "Quintication" method to obtain approximate analytic solutions of non-linear oscillators, Appl. Math. Comp. 243: 849 - 855.
Esmailzadeh, E; Younesian, D; Askari, H (2019). Analytical Methods in Nonlinear Oscillations: Approaches and Applications, Springer, Netherlands.

Ghalambaz, M; Ghalambaz, M; Edalatifar, M (2016). Nonlinear oscillation of nanoelectro-mechanical resonators using energy balance method: considering the size effect and the van der Waals force. Appl. Nanosci. 6: 309-317.

$\mathrm{He}$, JH (2006). Some asymptotic methods for strongly nonlinear equations. Int. J. Mod. Phy. B, 20(10), 1141-1199.

Ismail, GM (2017). An analytical coupled homotopyvariational approach for solving strongly nonlinear differential equation. J. Egypt. Math. Soc. 25(4): 434-437.

Ismail, GM; Abul-Ez, M; Farea, NM; Saad, N (2019). Analytical approximations to nonlinear oscillation of nanoelectro-mechanical resonators. Eur. Phy. J. Plus. 134: 47.

Sanchez, NE (2005). A view to the new perturbation technique valid for large parameters. J. Sound Vib. 282: 1309-1316. 